\title{
Safety and Diagnostic Efficacy of Gadobenate Dimeglumine in MRI of the Brain and Spine of Neonates and Infants
}

\author{
(DD.S. Enterline, (D).W Martin, (D) H.A Parmar, (D)F.M Triulzi, and (D) C. Colosimo
}

\begin{abstract}
BACKGROUND AND PURPOSE: Contrast-enhanced MR imaging provides essential information for pediatric imaging applications. We evaluated gadobenate dimeglumine for contrast-enhanced MR imaging of infants younger than 2 years of age.

MATERIALS AND METHODS: Ninety children younger than 2 years of age (including 55 children younger than 1 year) who underwent enhanced MR imaging of the CNS with gadobenate dimeglumine at $0.1 \mathrm{mmol} / \mathrm{kg}$ body weight $\pm 25 \%$ by volume were retrospectively enrolled at 2 imaging centers. Safety data were assessed for adverse events and, when available, vital signs and electrocardiogram and clinical laboratory values obtained from 48 hours before until 48 hours after the MR imaging examination. The efficacy of gadobenate dimeglumine-enhanced MR imaging was evaluated prospectively by 3 blinded, unaffiliated readers in terms of the accuracy of combined pre- and postcontrast images relative to precontrast images alone for differentiation of tumor from non-neoplastic disease and the correct diagnosis of specific disease. Differences were tested using the McNemar test. A possible effect of dose on diagnostic accuracy was assessed using the Fisher exact test.

RESULTS: Nine nonserious adverse events were reported for 8 (8.8\%) patients. Five adverse events occurred in patients 12 months of age or older. All events occurred at least 24 hours after gadobenate dimeglumine administration, and in each case, the investigating radiologist considered that there was no reasonable possibility of a relationship to gadobenate dimeglumine. No clinically meaningful changes in vital signs, electrocardiogram results, or laboratory parameters were reported. Accurate differentiation of tumor from non-neoplastic disease and exact matching of each specific MR imaging-determined diagnosis with the on-site final diagnosis were achieved in significantly more patients by each reader following evaluation of combined pre- and postcontrast images relative to precontrast images alone $(91.0 \%-94.4 \%$ versus $75.3 \%-87.6 \%, P<.04$, and $66.3 \%-73.0 \%$ versus $52.8 \%-58.4 \%, P<.02$, respectively). No significant differences $(P>.133)$ in diagnostic accuracy were noted between patients receiving $\leq 0.08 \mathrm{mmol} / \mathrm{kg}$ of gadobenate dimeglumine and patients receiving $>0.08 \mathrm{mmol} / \mathrm{kg}$ of gadobenate dimeglumine.
\end{abstract}

CONCLUSIONS: Gadobenate dimeglumine is safe and effective for pediatric MR imaging.

ABBREVIATIONS: $\mathrm{ECG}$ = electrocardiogram; $\mathrm{GBCA}$ = gadolinium-based contrast agent; $\mathrm{Gd}$ = gadolinium

$\mathbf{T}$

he choice of MR imaging contrast agents for use in pediatric subjects, particularly in neonates and infants younger than

Received July 26, 2019; accepted after revision September 18.

From the Department of Radiology (D.S.E.), Duke University, Durham, North Carolina; Department of Diagnostic Imaging (K.W.M.), University of California, San Francisco, Benioff Children's Hospital, Oakland, California; Department of Radiology (H.A.P.), University of Michigan, C.S. Mott Children's Hospital, Ann Arbor, Michigan; Department of Neuroradiology (F.M.T.), Fondazione Istituto di Ricovero e Cura a Carattere Scientifico Cà Granda Ospedale Maggiore Policlinic, Milan, Italy; and Radiodiagnostica e Neuroradiologia (C.C.), Institute of Radiology, Fondazione Policlinico Universitario 'A. Gemelli', Università Cattolica del Sacro Cuore, Rome, Italy. Grants from Bracco Diagnostics, specifically to enroll patients, were awarded to the University of California, San Francisco, CSF Benioff Children's Hospital in Oakland, California (Kenneth W. Martin) and the University of Michigan, C.S. Mott Children's Hospital in Ann Arbor, Michigan (Hemant A. Parmar).

Please address correspondence to David S. Enterline, MD, Duke University Medical Center, 3808 Erwin Rd, Durham, NC 27710; e-mail: david.enterline@duke.edu http://dx.doi.org/10.3174/ajnr.A6319
2 years of age, is increasingly driven by concerns over potential differences in safety among available gadolinium-based contrast agents (GBCAs). Early concern over the potential increased risk of nephrogenic systemic fibrosis in young children with immature renal function ${ }^{1-3}$ has been overshadowed in recent years by a perceived risk associated with gadolinium (Gd) retention following multiple exposures to certain types of GBCAs. ${ }^{4-7}$ Such concerns have led to a change in practice at some centers, with many departments opting to use macrocyclic GBCAs, which are considered more stable and, consequently, safer for potentially more vulnerable pediatric subjects. ${ }^{8}$ Recent evidence demonstrating T1 signal increases suggestive of Gd retention in the pediatric brain after the exclusive administration of macrocyclic GBCAs ${ }^{9}$ should encourage practitioners to look more closely at the available evidence and to select the most appropriate GBCA on the basis of 
not only the potential for Gd deposition but also diagnostic efficacy and other safety parameters such as the risk for immediate adverse events. ${ }^{10}$

Gadobenate dimeglumine (MultiHance; Bracco Diagnostics, Princeton, New Jersey) is a GBCA that has been proved safe among adult and pediatric (older than 2 years of age) subjects for a variety of indications. ${ }^{11-14}$ Compared with other GBCAs, gadobenate dimeglumine has 2 unique features that make it advantageous for contrast-enhanced MR imaging of children; on the one hand, it has increased relaxivity, ${ }^{15}$ which enables better depiction of very small or poorly enhancing lesions compared with other agents at equivalent doses, ${ }^{16}$ and on the other hand, it is partially eliminated (up to $5 \%$ of the injected dose) by the hepatobiliary route, rendering it suitable for both dynamic and delayed hepatobiliary phase imaging of the liver at a reduced dose. $^{17}$ In terms of the risk of nephrogenic systemic fibrosis, numerous studies point to its safety in patients with severely compromised renal function, ${ }^{18,19}$ and it is classified, together with the 3 macrocyclic agents, as a group II (lowest risk of nephrogenic systemic fibrosis) GBCA by the American College of Radiology. ${ }^{2}$ Studies of T1 signal changes in the pediatric brain following cumulative gadobenate dimeglumine exposure have revealed no differences relative to GBCA-naïve subjects in nonneurologic subjects (including in infants younger than 2 years of age at first exposure) who received between 5 and 15 doses of gadobenate dimeglumine at a dose of $0.05 \mathrm{mmol} / \mathrm{kg}$. ${ }^{20}$ Conversely, elevations of T1 signal were seen in 2 of 60 untreated (no radiochemotherapy) children with neurologic disease, but only after 16 and 20 injections of gadobenate dimeglumine at a higher dose of $0.1 \mathrm{mmol} / \mathrm{kg}^{21}$

A recent study in 200 pediatric hospital in-patients from 4 days to 15 years of age revealed an excellent safety profile for gadobenate dimeglumine with no adverse events or abnormal clinical laboratory values reported during the 24-hour monitoring period, even among patients who received several gadobenate dimeglumine exposures. ${ }^{14}$ Among these patients were 57 who were 2 years of age or younger, of whom 47 were 1 year of age or younger at first exposure (including 18 that were 1 month or younger). Gadobenate dimeglumine is currently approved in Europe for liver imaging and in the United States and elsewhere for a range of indications, including enhanced MR imaging of the CNS in children younger than 2 years of age. ${ }^{22}$ The present study was performed to further evaluate the safety and efficacy of gadobenate dimeglumine in neonates and infants 2 years of age or younger referred for routine diagnostic MR imaging of the CNS.

\section{MATERIALS AND METHODS}

This was a retrospective evaluation of safety data for patients 2 years of age or younger with suspected or known disease of the CNS who had undergone contrast-enhanced MR imaging with gadobenate dimeglumine as part of the clinical work-up. An additional prospectively designed blinded read of images from these patients was performed to confirm the efficacy of gadobenate dimeglumine. Patients underwent imaging at 2 centers (Benioff Children's Hospital, Oakland, California; C.S. Mott Children's Hospital, Ann Arbor, Michigan) in the United States between June 12, 2008, and December 5, 2014. The study was conducted in accordance with the International Congress on Harmonization, Good Clinical Practice, US FDA regulations, and ethical principles outlined in the Declaration of Helsinki and all applicable local regulations. The study protocol was reviewed and approved by the institutional review board at each center, was compliant with the Health Insurance Portability and Accountability Act, and was registered at www. clinicaltrials.gov.

\section{Patients}

Male and female patients were eligible for inclusion if they were 2 years of age or younger, received gadobenate dimeglumine at a documented nominal dose of $0.1 \mathrm{mmol} / \mathrm{kg}$ body weight $( \pm 25 \%$ in volume administered) for known or highly suspected enhancing disease of the brain or spine, and had complete demographic and safety data available. Eligible patients were included consecutively from a prospectively defined start date (December 5, 2014) and continued chronologically backward in strict order until the prospectively designed enrollment was attained. A prospective, blinded assessment of efficacy was then performed for all patients who were eligible for the safety evaluation for whom pre- and postdose T1-weighted spin-echo/FSE and/or gradient recalledecho images as well as T2-weighted spin-echo/FSE and FLAIR (if acquired) images were available.

\section{MR Imaging}

MR imaging was performed at 1.5T (Ingenia and Gyroscan Intera; Philips Healthcare, Best, the Netherlands) for all patients apart from 7 subjects whose images were acquired at 3T (Ingenia and Achieva; Philips Healthcare). A standard protocol comprising T1-weighted spin-echo, T1-weighted gradient recalled-echo, T2-weighted FSE, and T2-weighted FLAIR acquisitions before contrast injection, and T1-weighted spin-echo and T1-weighted gradient recalled-echo acquisitions after injection ensured protocol uniformity across the 2 sites and in individual patients. Gadobenate dimeglumine was administered manually as a nominal bolus dose of $0.1 \mathrm{mmol} / \mathrm{kg}$ body weight $(0.2 \mathrm{~mL} / \mathrm{kg})$. However, due to the retrospective nature of the study, a dose of $0.1 \mathrm{mmol} / \mathrm{kg} \pm 25 \%$ by volume was prospectively defined for patient inclusion.

\section{Safety Assessments}

Safety data recorded by the investigating radiologist at the time of the examination were evaluated in terms of clinical adverse events and, when available, vital signs and electrocardiogram (ECG) and clinical laboratory determinations. An adverse event was defined as any untoward medical occurrence during the timeframe associated with the administration of gadobenate dimeglumine that did not necessarily need to be causally related to the administration of gadobenate dimeglumine. If an adverse event was considered related to sedation or anesthesia, this was recorded as part of the event description.

All safety data obtained from 48 hours before until 48 hours after the examination were recorded on an individual, patientspecific case report form. Adverse events with unknown onset times were counted beginning during the timeframe associated with gadobenate dimeglumine administration. If laboratory data 
Table 1: Patient demographic characteristics and details regarding type of MR examination, exposure to gadobenate dimeglumine, and sedation during examination

\begin{tabular}{|c|c|c|c|c|c|}
\hline Demographic & Overall & 0 to $<1$ Month & 1 to $<6$ Months & 6 to $<12$ Months & 12-24 Months \\
\hline No. of subjects & 90 & 13 & 23 & 19 & 35 \\
\hline Male/female & $48: 42$ & $4: 9$ & 11:12 & $16: 3$ & $17: 18$ \\
\hline \multicolumn{6}{|l|}{ Age (mo) } \\
\hline Mean & $9.7 \pm 7.5$ & $0.3 \pm 0.3$ & $3.0 \pm 1.3$ & $9.1 \pm 1.8$ & $17.9 \pm 3.1$ \\
\hline Range & $0.1-23.3$ & $0.1-0.8$ & $1.1-5.6$ & $6.0-11.5$ & $12.2-23.3$ \\
\hline \multicolumn{6}{|l|}{ Weight (Kg) } \\
\hline Mean & $7.9 \pm 3.3$ & $3.5 \pm 0.7$ & $5.4 \pm 1.5$ & $8.6 \pm 1.8$ & $10.8 \pm 2.0$ \\
\hline Range & $2.3-16.3$ & $2.3-4.8$ & $2.6-9.6$ & $5.2-12.1$ & $7.4-16.3$ \\
\hline \multicolumn{6}{|l|}{ Height $(\mathrm{cm})$} \\
\hline Mean & $68.4 \pm 13.5$ & $46.4 \pm 6.7$ & $59.4 \pm 6.4$ & $68.7 \pm 5.2$ & $81.0 \pm 5.8$ \\
\hline Range & $32-91$ & $32-51$ & $47-71$ & 61-76 & 70-91 \\
\hline \multicolumn{6}{|c|}{ Type of examination } \\
\hline Brain & 83 & 12 & 23 & 17 & 31 \\
\hline Spine & 7 & 1 & 0 & 2 & 4 \\
\hline \multicolumn{6}{|l|}{ Dose (mmol/kg) } \\
\hline Mean & $0.084 \pm 0.015$ & $0.092 \pm 0.025$ & $0.089 \pm 0.018$ & $0.085 \pm 0.01$ & $0.078 \pm 0.006$ \\
\hline Range & $0.07-0.13$ & $0.07-0.13$ & $0.07-0.13$ & $0.07-0.10$ & $0.07-0.10$ \\
\hline \multicolumn{6}{|c|}{ Sedation/anesthesia ${ }^{a}$} \\
\hline Yes & 72 & 7 & 16 & 15 & 34 \\
\hline No & 7 & 3 & 3 & 1 & 0 \\
\hline NA & 11 & 3 & 4 & 3 & 1 \\
\hline
\end{tabular}

Note:-NA indicates not available.

a Patients may have received $>1$ drug for sedation

were available, the normal ranges for the parameters measured were included. The last laboratory measurement before the administration of gadobenate dimeglumine was taken as the baseline value.

\section{Efficacy Assessments}

MR images were evaluated by 3 independent neuroradiologists (C.C., D.S.E., and F.M.T.; each with $>20$ years' experience) who were unaffiliated with the enrollment centers and blinded to all patient clinical profiles. Evaluation was performed using the thin client of the AquariusNet server (Version 4.4.5.36; TeraRecon, San Mateo, California). Blinded reads consisted of independent assessments of precontrast images and combined pre- and postcontrast images in a randomized order.

Patient diagnoses were determined at the disease (tumor versus non-neoplastic) level and at specific diagnosis levels from a list of 54 coded diagnoses covering brain and spinal diseases of different natures (including neoplastic, inflammatory, degenerative, vascular, and postoperative conditions). Diagnoses by the blinded readers were subsequently matched with the final on-site patient diagnosis which, in each case, was determined by the investigating radiologist on the basis of all available clinical, pathologic, radiologic, and surgical information.

Finally, each reader was asked whether the postcontrast images provided additional information over precontrast images based on applicable categories described elsewhere. ${ }^{23}$

\section{Statistical Analysis}

Statistical analyses were performed using SAS software (Version 9.3; SAS Institute, Cary, North Carolina). Continuous measurements were reported as mean $\pm \mathrm{SD}$, while categoric assessments were described as number (\%). The final diagnosis made at each investigator site was considered the reference standard. Diagnostic accuracy on precontrast and combined pre- and postcontrast images was estimated for each reader for both the differentiation of tumor from non-neoplastic disease and the correct diagnosis at specific disease level. The McNemar test was used to test the difference in diagnostic accuracy between precontrast and combined pre- and postcontrast image sets by reader. The Fisher exact test was used to test the difference in diagnostic accuracy for combined pre- and postcontrast image sets for contrast doses of $\leq 0.08 \mathrm{mmol} / \mathrm{kg}$ body weight and $>0.08 \mathrm{mmol} /$ $\mathrm{kg}$ body weight.

Assuming an expected difference in accuracy between precontrast and combined pre- and postcontrast image sets of $10 \%$ with $12 \%$ discordant pairs, we calculated a sample size of 90 patients to provide $>85 \%$ power for a McNemar test of equality in accuracy.

\section{RESULTS}

Ninety patients meeting the inclusion criteria were included in the safety evaluation. Of these 90 patients, 89 (98\%) had pre- and postdose efficacy data available and were included in the prospective blinded evaluation of gadobenate dimeglumine efficacy. One patient 13 months of age was excluded from the efficacy evaluation due to image acquisitions occurring on different days, with different equipment, and in different planes.

Demographic details for the 90 patients evaluated for safety are shown in Table 1. Patient ages ranged from 0.1 month ( 2 days postnatal) to 23.3 months ( 23 months, 7 days). The age distribution included 35 (39\%) between 12 and younger than 24 months; 19 (21\%) between 6 and younger than 12 months, 23 (26\%) between 1 and younger than 6 months; and 13 (14\%) younger than 1 month. An MR imaging examination of the brain was performed in 83 (92.2\%) cases, while MR imaging of the spine was performed in 7 (7.8\%) cases.

A summary of relevant medical history is given in Table 2. At least 1 finding was reported for 71 (78.9\%) patients. The most 
commonly reported medical history findings were oncologic, occurring in 23 of the 71 patients with available information.

Serum creatinine values measured before and in close temporal proximity to the administration of gadobenate dimeglumine were available for 61 patients (Table 3). Estimated glomerular filtration rate values calculated using the Schwartz formula for patients younger than 2 years of age ${ }^{24}$ were reported for 40 patients with available height data. Mean and median values across the age groups were consistent with those reported for children with normal age-related renal function; immaturity of clearance was observed in infants younger than 1 month and a gradual decline of serum creatinine levels was observed during the first year, reflecting normal kidney maturation and muscle mass accretion. ${ }^{25}$

Administration of sedation and/or anesthesia before the examination was reported for $72(80 \%)$ patients. Seven patients did not receive sedation or anesthesia before the examination. No information could be retrieved from clinical records for 11 subjects. Propofol, alone or in combination with other drugs, including sevoflurane, isoflurane, fentanyl, midazolam, nitrous oxide, succinylcholine, vecuronium bromide, and cisatracurium besilate, was administered to 56/72 (77.8\%) patients without complications.

The distribution of gadobenate dimeglumine doses is presented in Table 4. Overall, 52/89 (58.4\%) patients received a dose of $\leq 0.08 \mathrm{mmol} / \mathrm{kg}$ body weight $(0.065-0.08 \mathrm{mmol} / \mathrm{kg})$, while $37 /$ $89(41.6 \%)$ patients received a dose of $>0.08 \mathrm{mmol} / \mathrm{kg}$ body weight $(0.082-0.132 \mathrm{mmol} / \mathrm{kg})$. Gadobenate dimeglumine was administered in accordance with the standard practice at each investigating center.

Table 2: Relevant medical history of evaluated patients ${ }^{\mathrm{a}}$

\begin{tabular}{lc}
\multicolumn{1}{c}{ History } & \\
\hline No. of patients included & $n=90$ \\
No. of patients with at least 1 finding & $71(78.9)$ \\
No. of patients by finding & \\
Oncology & $23(25.6)$ \\
Metabolic & $1(1.1)$ \\
Congenital & $6(6.7)$ \\
Other & $48(53.3)$ \\
Cerebrovascular & $23(25.6)$ \\
Hemorrhagic & $3(3.3)$ \\
Infarct & $3(3.3)$ \\
Ischemic & $13(14.4)$ \\
Vascular & $4(4.4)$ \\
Hydrocephalus & $3(3.3)$ \\
Inflammatory/Infectious & $15(16.7)$ \\
Injury & $7(7.8)$ \\
Seizure & $4(4.4)$ \\
Other findings & $6(6.7)$ \\
\hline
\end{tabular}

a Numbers add up to $>100 \%$ because a patient may have findings in $>1$ category. Data in parentheses are percentages of total $[n=90]$ patients.

\section{Safety Findings}

Adverse Events. Nine nonserious adverse events were reported for $8(8.8 \%)$ patients. Six adverse events were reported in 5 patients 12 months of age or older. Three adverse events $(2$ cases of fever, 1 of poor urine output) were considered mild, and 6 (malignant hyperthermia, 3 cases of seizures/seizurelike activity, hypokinesis, decreased responsiveness) were moderate in intensity. All events occurred at or $>24$ hours after gadobenate dimeglumine administration, and in all cases, the investigating radiologist considered that there was no reasonable possibility of a relationship to the administration of gadobenate dimeglumine. All patients recovered, though 1 patient recovered with sequelae following an operation.

Vital Signs. Vital signs were monitored at standard intervals for all sedated patients (52/90 [57.8\%]) but were not routinely monitored for nonsedated patients. No clinically meaningful changes were recorded by the investigators.

ECGs. ECG data from at least 1 postdose time point were available for 48/90 (53.3\%) patients. No clinically meaningful changes from baseline were observed in any patient with data available.

Clinical Laboratory Values. Data for hematology and clinical chemistry at pre- and postdose were available for $47 / 90$ (52.2\%) patients. On the basis of available data, no clinically meaningful changes from baseline in any hematology or clinical chemistry parameter were reported for any patient.

\section{Efficacy Results}

Accurate differentiation of neoplastic from non-neoplastic disease was achieved by all 3 blinded readers in significantly $(P<.05)$ more patients following evaluation of combined preand postcontrast images relative to precontrast images alone (91.0\%-94.4\% versus $75.3 \%-87.6 \%$, Table 5). Three-reader agreement for the differential diagnosis was achieved for 63/89 (70.8\%) patients based on precontrast images alone, but for $77 / 89$ (86.5\%) patients based on the evaluation of combined pre- and postcontrast images.

Similar results were obtained when each reader was asked to ascribe a specific lesion diagnosis from a list of 54 coded diagnoses. Each reader assigned a correct diagnosis relative to the onsite final clinical diagnosis in significantly $(P \leq .02)$ more patients based on assessment of combined pre- and postcontrast images relative to precontrast images alone $(66.3 \%-73.0 \%$ versus $52.8 \%$ $58.4 \%$, Table 5). No reader provided an accurate diagnosis for $22 /$ $89(24.7 \%)$ patients based on the evaluation of precontrast images alone. The number of patients for whom an accurate diagnosis

Table 3: Serum creatinine values

\begin{tabular}{|c|c|c|c|c|c|}
\hline Demographic & Overall & 0 to $<1$ Month & 1 to $<6$ Months & 6 to $<12$ Months & 12-24 Months \\
\hline $\begin{array}{l}\text { No. of subjects } \\
\mathrm{sCr}(\mathrm{mg} / \mathrm{mL})\end{array}$ & 61 & 12 & 16 & 11 & 22 \\
\hline Mean & $0.3 \pm 0.19$ & $0.5 \pm 0.23$ & $0.3 \pm 0.25$ & $0.2 \pm 0.07$ & $0.3 \pm 0.07$ \\
\hline $\begin{array}{l}\text { Range } \\
\text { eGFR }(\mathrm{mL} / \mathrm{min} / 1.73 \mathrm{r}\end{array}$ & $0.1-1.2$ & $0.2-0.9$ & $0.1-1.2$ & $0.1-0.4$ & $0.2-0.4$ \\
\hline Mean & $131.7 \pm 60.3$ & $59.3 \pm 31.7$ & $92.4 \pm 43.2$ & $150.4 \pm 18.3$ & $176.3 \pm 47.2$ \\
\hline Range & $17.6-255.6$ & $24.0-114.8$ & $17.6-153.9$ & $114.0-168.8$ & $99.0-255.6$ \\
\hline
\end{tabular}

Note:- $\mathrm{SCr}$ indicates serum creatinine; eGFR, estimated glomerular filtration rate. 
was not made was lower $(17 / 89 ; 19.1 \%)$ when assessment was made of combined pre- and postcontrast images. Agreement on an accurate specific diagnosis by all 3 readers was achieved for $29 / 89$ (32.6\%) patients based on precontrast images alone but for $50 / 89$ (56.2\%) patients when evaluation was made of combined pre- and postcontrast images.

No differences in either the differential diagnosis (neoplasticversus-non-neoplastic disease, $P<.133)$ or a specific disease diagnosis $(P>.344)$ were noted by any reader for patients who received a gadobenate dimeglumine dose of $\leq 0.08 \mathrm{mmol} / \mathrm{kg}$ body weight compared with patients who received a dose of $>0.08 \mathrm{mmol} / \mathrm{kg}$ body weight (Table 6 ).

The presence and pattern of contrast enhancement were considered radiologically helpful in 78/89 (87.6\%), 85/89 (95.5\%), and $57 / 89(64 \%)$ patients by readers 1,2 , and 3 , respectively. The primary benefit was considered the improvement in lesion conspicuity as defined by an improved visualization of the size, extent, and/or margins of a lesion or better prediction of the grade, histologic type, vascularity, and/or aggressiveness of a lesion (Figs 1-3). Lack of enhancement was also recognized as a potentially useful factor in image interpretation but in a smaller percentage of patients $(6.0 \%, 16.8 \%$, and $4.5 \%$; readers 1,2 , and 3 , respectively).

Table 4: Gadobenate dimeglumine dose distribution

\begin{tabular}{lcc}
\hline \multicolumn{2}{c}{ Volume/Dose Classes } & \\
\cline { 1 - 2 } $\begin{array}{l}\text { Volume Administered } \\
(\mathrm{mL} / \mathrm{kg})\end{array}$ & $\begin{array}{c}\text { Dose Administered } \\
\text { (mmol/kg) }\end{array}$ & $\begin{array}{c}\text { No. of } \\
\text { Patients }\end{array}$ \\
\hline$\leq 0.14$ & $\leq 0.07$ & 2 \\
$0.142-0.15$ & $0.071-0.075$ & 25 \\
$0.152-0.16$ & $0.076-0.08$ & 25 \\
$0.162-0.18$ & $0.081-0.09$ & 20 \\
$0.182-0.2$ & $0.091-0.1$ & 7 \\
$0.202-0.264$ & $0.101-0.132$ & 11 \\
Total & & 90 \\
\hline
\end{tabular}

\section{DISCUSSION}

Whereas the accumulated experience in MR imaging of the CNS of adult patients is helpful for imaging the pediatric population, pediatric imaging presents unique challenges in terms of approaches to image acquisition and, in particular, concerns about patient safety. ${ }^{26,27}$ Nevertheless, despite numerous recent studies that have focused on potential safety issues associated with GBCA use in the pediatric population, ${ }^{1,3-9}$ little, if any, evidence of increased risk due to GBCA administration has been demonstrated in the roughly 30 years since the approval of the first GBCA. As with all contrast-enhanced studies, the fear of adverse consequences, no matter how rare the event or unfounded the fear, should be off-set against the clinically relevant diagnostic information available from the examination. In the case of pediatric neuroimaging, GBCA-enhanced MR imaging provides essential information on a variety of conditions, including congenital malformations, tumors, infections, metabolic disorders, epilepsy, and inflammatory and cerebrovascular diseases. Postcontrast imaging provides detailed information on the location, type, and number of lesions that are essential for diagnosis and treatment planning.

The safety and efficacy profiles of GBCAs in children 2 years of age or older are relatively well-established, in part because most GBCAs have regulatory approval for children 2 years of age or older. Much more limited information is available for children younger than 2 years of age. One of the principal concerns relating to young children, and particularly neonates, is that the hepatic and renal clearance mechanisms are relatively underdeveloped compared with those in adults. ${ }^{28,29}$ In full-term infants, glomerular filtration efficiency roughly equivalent to adult levels does not occur until about 6 months. ${ }^{30}$ Nevertheless, pharmacokinetic studies of renally excreted drugs, including GBCAs, in healthy neonates have revealed kinetic behavior comparable with that in older children and adults. ${ }^{30,31}$

Our study revealed no evidence of any adverse events attributable to gadobenate dimeglumine administration in children younger than 2 years of age, even among the youngest children

Table 5: Evaluation of diagnostic performance on combined pre- and postcontrast MR images relative to precontrast images alone

\begin{tabular}{|c|c|c|c|c|}
\hline End Point & Reader & Precontrast $(n=89)$ & Pre- + Postcontrast $(n=89)$ & $P$ Value $^{\mathrm{a}}$ \\
\hline \multirow[t]{3}{*}{ Tumor vs nontumor } & 1 & $75(84.3 \%)$ & $84(94.4 \%)$ & .003 \\
\hline & 2 & $67(75.3 \%)$ & $81(91.0 \%)$ & $<.001$ \\
\hline & 3 & $78(87.6 \%)$ & $82(92.1 \%)$ & .046 \\
\hline \multirow[t]{3}{*}{ Specific diagnosis } & 1 & $52(58.4 \%)$ & 65 (73.0\%) & .003 \\
\hline & 2 & $47(52.8 \%)$ & $63(70.8 \%)$ & $<.001$ \\
\hline & 3 & $50(56.2 \%)$ & $59(66.3 \%)$ & .02 \\
\hline
\end{tabular}

${ }^{\mathrm{a}}$ McNemar test.

Table 6: Comparison of diagnostic performance on combined pre- and postcontrast images in patients administered gadobenate dimeglumine at doses of $\leq 0.08 \mathrm{mmol} / \mathrm{kg}$ body weight compared with doses $>0.08 \mathrm{mmol} / \mathrm{kg}$ body weight

\begin{tabular}{ccccc}
\hline End Point & Reader & $\leq \mathbf{0 . 0 8} \mathbf{~ m m o l} / \mathbf{k g}(\boldsymbol{n}=\mathbf{5 2})$ & $\mathbf{> 0 . 0 8} \mathbf{~ m m o l} / \mathbf{k g}(\boldsymbol{n}=\mathbf{3 7})$ & \multicolumn{1}{c}{$\boldsymbol{P}$ Value } \\
\hline Tumor vs nontumor & 1 & $50(96.2 \%)$ & $34(91.9 \%)$ & .645 \\
& 2 & $45(86.5 \%)$ & $36(97.3 \%)$ & .133 \\
& 3 & $47(90.4 \%)$ & $35(94.6 \%)$ & .695 \\
Specific diagnosis & 1 & $40(76.9 \%)$ & $25(67.6 \%)$ & .344 \\
& 2 & $38(73.1 \%)$ & $25(67.6 \%)$ & .64 \\
& 3 & $35(67.3 \%)$ & $24(64.9 \%)$ & .824 \\
\hline
\end{tabular}

${ }^{a}$ Fisher exact test. 

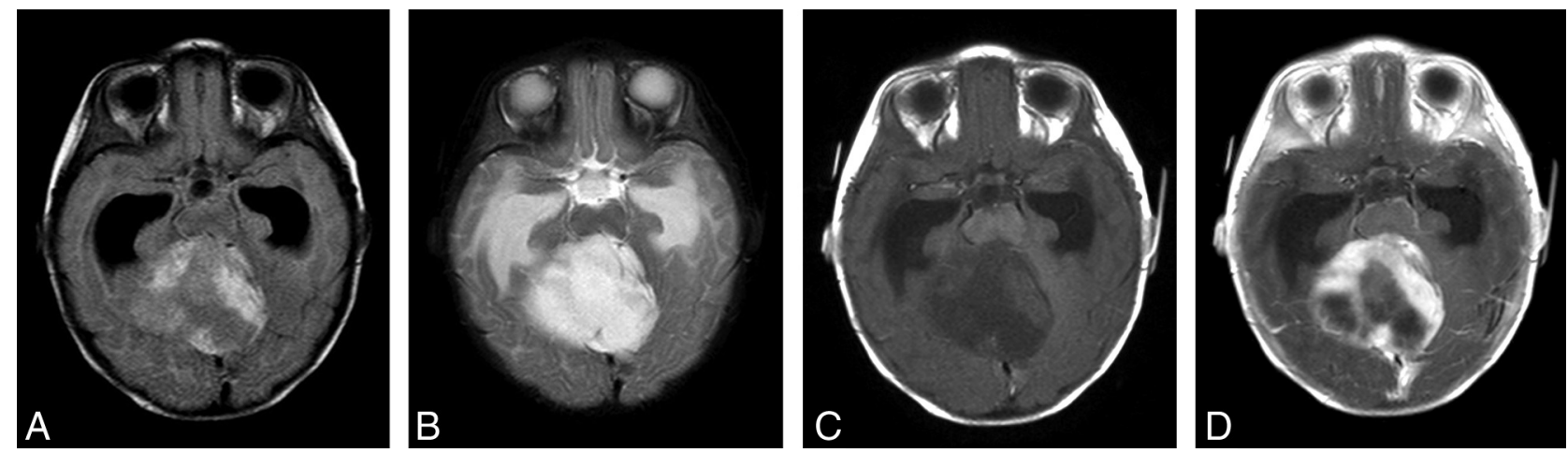

FIG 1. MR imaging in a 4.3-month-old infant with cerebellar pilocytic astrocytoma shows a large intra-axial mass, which is hyperintense on FLAIR $(A)$ and T2-weighted $(B)$ images with surrounding edema. The lesion is hypointense on the T1-weighted precontrast image $(C)$ and shows heterogeneous-but-avid postcontrast enhancement $(D)$ after contrast administration $(0.08 \mathrm{mmol} / \mathrm{kg}$ of gadobenate dimeglumine $[1 \mathrm{~mL}]$ administered as a manual bolus). Also note marked obstructive hydrocephalus with enlarged bilateral temporal horns.
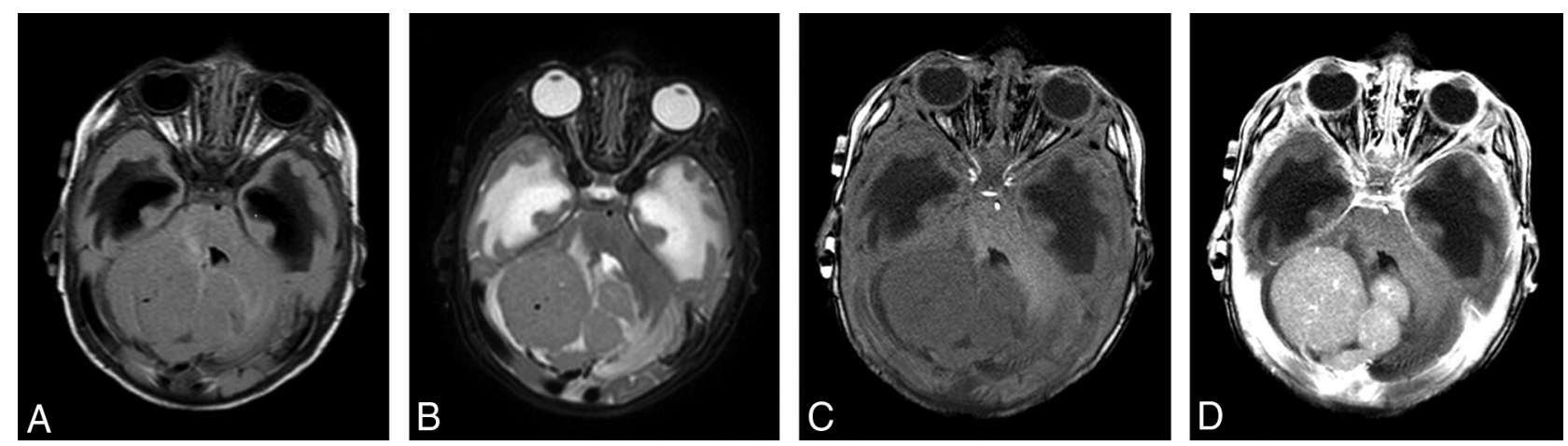

FIG 2. MR imaging in a 2.3-month-old infant with desmoplastic medulloblastoma shows a large multinodular intra-axial mass, which is isointense on FLAIR $(A)$ and T2-weighted $(B)$ images with mild surrounding edema. The lesion is slightly hypointense on the T1-weighted precontrast image $(C)$ and shows moderate homogeneous enhancement $(D)$ after contrast administration $(0.07 \mathrm{mmol} / \mathrm{kg}$ of gadobenate dimeglumine, $0.8 \mathrm{~mL}$ administered as a manual bolus). Also noted is obstructive hydrocephalus with extensive periventricular interstitial edema.
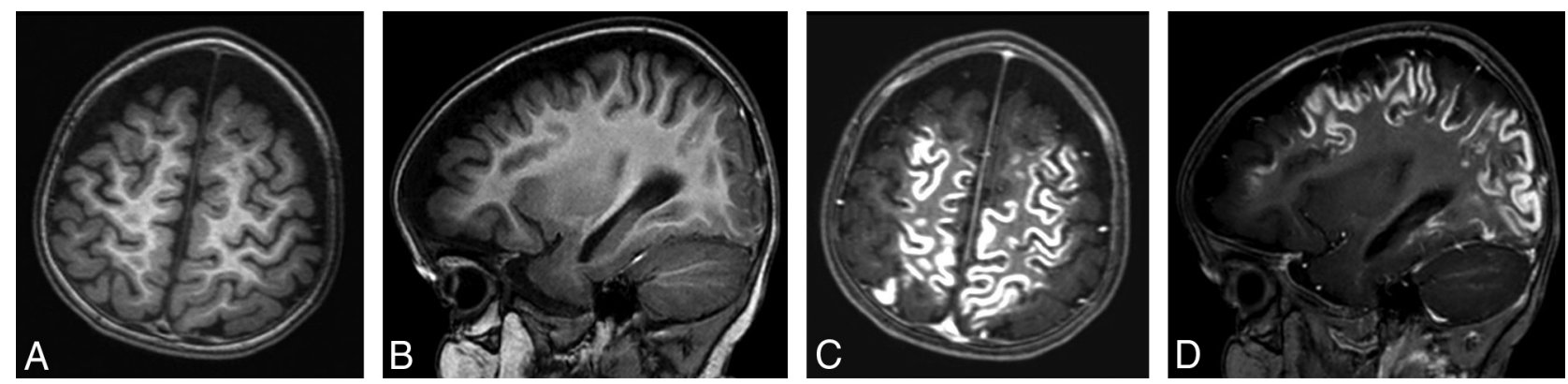

FIG 3. MR imaging in a 15-month-old girl with known subacute infarctions. Axial and sagittal T1-weighted images acquired before ( $A$ and $B$ ) and after $(C$ and $D)$ contrast administration $(0.08 \mathrm{mmol} / \mathrm{kg}$ gadobenate dimeglumine $[1.5 \mathrm{~mL}]$ administered as a manual bolus). Extensive gyral enhancement in the cerebral hemispheres is clearly demonstrated on postcontrast images.

evaluated. Overall, just 9 adverse events were reported, each of which occurred at least 24 hours after gadobenate dimeglumine administration and none of which were considered attributable to the administration of gadobenate dimeglumine. In terms of the safety profile, our findings are in agreement with those of Schneider et al, ${ }^{14}$ who evaluated gadobenate dimeglumine safety in 200 pediatric patients from 4 days to 15 years of age (including 47 patients 1 year of age or younger). In their study, no adverse events were reported after the administration of up to 10 injections of gadobenate dimeglumine, and no changes in creatinine or bilirubin levels were noted, even among the very youngest children.

In common with the study by Schneider et al, ${ }^{14}$ approximately $80 \%$ of our patients were imaged under heavy sedation or general anesthesia. The need for sedation reflects the need for the child to lie still in the magnet for a relatively long time. One benefit of 
sedation in very young children is that changes in vital signs, ECG traces, and even laboratory measurements from acquired blood samples can more readily be assessed objectively. In our study, as in the study by Schneider et al, ${ }^{14}$ no clinically meaningful changes from baseline were noted for any patient for ECG intervals, vital signs, or laboratory values. Our results also compare very favorably with safety findings reported for the macrocyclic GBCAs gadoterate meglumine ${ }^{32,33}$ and gadobutrol. ${ }^{31,34}$ Those studies similarly reported no, ${ }^{33,34}$ or at most $1,{ }^{31,32}$ adverse event in children younger than 2 years of age that was considered related to the administration of GBCA.

In terms of diagnostic efficacy, gadobenate dimeglumine has proved efficacious in pediatric subjects not only for body applications ${ }^{14}$ but also specifically for brain tumor imaging. ${ }^{16}$ Our findings confirm those of previous studies in demonstrating excellent accuracy ( $>91 \%$ for all 3 readers) for the differentiation of neoplastic from non-neoplastic disease. Most important, the distribution of neoplastic-versus-non-neoplastic disease in the patients' diagnoses was balanced in our study (57.3\% versus $42.3 \%$, respectively), with roughly equivalent representation of both intra- and extra-axial tumors in patients with neoplasms. Regarding the more challenging assessment of accuracy for a specific diagnosis, values from $66.3 \%$ to $73.0 \%$ were obtained for the 3 blinded readers. These values should be considered very good, given the fully blinded and independent reading conditions under which the images were evaluated. It is well-established that knowledge of clinical data significantly improves lesion diagnosis for other contrastenhanced MR imaging applications ${ }^{35}$ and thus it is to be expected that more correct specific diagnoses would have been made in this study had the readers been aware of the medical history and clinical characteristics of the patients.

Compared with other GBCAs, gadobenate dimeglumine has considerably higher $\mathrm{r} 1$ relaxivity, ${ }^{15}$ which translates into significantly better diagnostic performance for brain tumor imaging when administered at a dose equivalent to that of a comparator GBCA. $^{36,37}$ Recent studies have highlighted the value of this higher $\mathrm{r} 1$ relaxivity in permitting lower gadobenate dimeglumine doses without the loss of diagnostic information relative to that attained with a comparator GBCA at higher dose. ${ }^{37-41}$ Our evaluation revealed no significant differences between doses lower and higher than $0.08 \mathrm{mmol} / \mathrm{kg}$ body weight for either differential or specific disease diagnoses. These findings lend support to previous studies that have looked at reduced doses of gadobenate dimeglumine for neuroimaging applications ${ }^{37-41}$ and suggest that a gadobenate dimeglumine dose lower than the standard GBCA dose $(0.1 \mathrm{mmol} / \mathrm{kg}$ body weight $)$ may be appropriate for neuroimaging in many cases. Notably, the gadobenate dimeglumine dose approved by the US FDA for enhanced MR imaging of the CNS in children younger than 2 years of age is $0.1-0.2 \mathrm{~mL} / \mathrm{kg}$ body weight, corresponding to $0.05-0.1 \mathrm{mmol} / \mathrm{kg}$ body weight. ${ }^{22}$

Such dose-lowering opportunities might be considered highly clinically relevant, given the current concern over T1 hyperintensity and gadolinium retention following GBCA administration. In this regard, a study in pediatric non-neurologic subjects who received between 5 and 15 doses of 0.05 $\mathrm{mmol} / \mathrm{kg}$ of gadobenate dimeglumine revealed no evidence of T1 hyperintensity in the dentate nucleus or globus pallidus compared with age- and weight-matched control subjects who had never been administered any GBCA. ${ }^{20}$ Conversely, a study in neurologic patients revealed T1 hyperintensity in 2 of 60 pediatric subjects who received 16 and 20 doses of $0.1 \mathrm{mmol} / \mathrm{kg}$ of gadobenate dimeglumine. ${ }^{21}$ Whether the T1 signal changes noted in these 2 subjects reflected the higher dose used $(0.1$ versus $0.05 \mathrm{mmol} / \mathrm{kg}$ ) or whether they were a consequence of the different underlying clinical condition of the patients (neurologic versus non-neurologic) is unclear and should be the subject of further study. However, the 16 and 20 doses given to these 2 patients were the highest cumulative doses across the entire patient cohort. ${ }^{21}$ It should be noted that T1 signal changes in the pediatric brain have also been seen after the exclusive administration of macrocyclic GBCAs, ${ }^{9}$ and that confirmed gadolinium presence has been reported in the brains of postmortem subjects even after the administration of single doses of macrocyclic GBCA. ${ }^{42}$

Most important, no clinical manifestations or adverse clinical outcomes related to $\mathrm{T} 1$ signal changes or brain Gd retention have been observed at this time following the repeat administration of any GBCA, and there is no evidence of any effect on cognitive or neurobehavioral development, as also recognized by the US FDA. Although studies to assess the impact of cumulative GBCA administration on human development and neurologic function are difficult to perform, studies in animals, which are invariably taken as indicative of the human situation, have revealed no impact of gadobenate dimeglumine on growth, maturation, behavior, or cognitive function of neonatal and juvenile rats, even after very high cumulative doses $(15 \mathrm{mmol} / \mathrm{kg}$; corresponding to about 25 injections of a standard $0.1-\mathrm{mmol} / \mathrm{kg}$ dose in humans). ${ }^{43}$

A limitation of our study was its retrospective design. However, whereas patients were enrolled retrospectively at each of the 2 sites, patient inclusion began from a prospectively defined start date and continued chronologically backward in strict order until the planned enrollment was attained. This enrollment procedure ensured an unbiased approach to patient inclusion. Furthermore, image assessment was performed in a prospective manner by 3 independent readers who were unaffiliated with the enrollment centers and fully blinded to all patient information.

\section{CONCLUSIONS}

Our findings confirm that contrast-enhanced MR imaging of the CNS with gadobenate dimeglumine at a dose between 0.065 and $0.132 \mathrm{mmol} / \mathrm{kg}$ body weight is safe, well-tolerated, and effective in patients younger than 2 years of age. The potential to lower the administered dose because of the higher $\mathrm{r} 1$ relaxivity of gadobenate dimeglumine may be considered beneficial in pediatric patients, particularly considering the cumulative nature of gadolinium retention in the brain.

Disclosures: David S. Enterline_-UNRELATED: Board Membership: Bracco Diagnostics, Comments: Scientific Advisory Board; Consultancy: Bracco Diagnostics. Kenneth W. Martin—RELATED: Grant. Children's Hospital and Research Center Oakland.* Hemant 
A. Parmar-RELATED: Other: Bracco Diagnostics, Comments: research grant to enroll study patients.* Cesare Colosimo—UNRELATED: Consultancy: Bracco Diagnostics, Comments: $€ 5000$ as a blinded reader for evaluating MR images of the enrolled patients. *Money paid to the institution.

\section{REFERENCES}

1. Nardone B, Saddleton E, Laumann AE, et al. Pediatric nephrogenic systemic fibrosis is rarely reported: a RADAR report. Pediatr Radiol 2014;44:173-80 CrossRef Medline

2. American College of Radiology. ACR Manual on Contrast Media. Version 10.3. Reston: American College of Radiology; 2017

3. Bedoya MA, White AM, Edgar JC, et al. Effect of intravenous administration of contrast media on serum creatinine levels in neonates. Radiology 2017;284:530-40 CrossRef Medline

4. Roberts DR, Chatterjee AR, Yazdani M, et al. Pediatric patients demonstrate progressive T1-weighted hyperintensity in the dentate nucleus following multiple doses of gadolinium-based contrast agent. AJNR Am J Neuroradiol 2016;37:2340-47 CrossRef Medline

5. $\mathrm{Hu} \mathrm{HH}$, Pokorney A, Towbin $\mathrm{RB}$, et al. Increased signal intensities in the dentate nucleus and globus pallidus on unenhanced T1weighted images: evidence in children undergoing multiple gadolinium MRI exams. Pediatr Radiol 2016;46:1590-98 CrossRef Medline

6. Flood TF, Stence NV, Maloney JA, et al. Pediatric brain: repeated exposure to linear gadolinium-based contrast material is associated with increased signal intensity at unenhanced T1-weighted MR imaging. Radiology 2017;282:222-28 CrossRef Medline

7. Radbruch A, Haase R, Kickingereder P, et al. Pediatric brain: no increased signal intensity in the dentate nucleus on unenhanced T1-weighted MR images after consecutive exposure to a macrocyclic gadolinium-based contrast agent. Radiology 2017;283:828-36 CrossRef Medline

8. Blumfield E, Moore MM, Drake MK, et al. Survey of gadoliniumbased contrast agent utilization among the members of the Society for Pediatric Radiology: a Quality and Safety Committee report. Pediatr Radiol 2017;47:665-73 CrossRef Medline

9. Rossi Espagnet MC, Bernardi B, Pasquini L, et al. Signal intensity at unenhanced T1-weighted magnetic resonance in the globus pallidus and dentate nucleus after serial administrations of a macrocyclic gadolinium-based contrast agent in children. Pediatr Radiol 2017;47:1345-52 CrossRef Medline

10. Gulani V, Calamante F, Shellock FG, et al; International Society for Magnetic Resonance in Medicine. Gadolinium deposition in the brain: summary of evidence and recommendations. Lancet Neurol 2017;16:564-70 CrossRef Medline

11. Kirchin MA, Pirovano G, Venetianer C, et al. Safety assessment of gadobenate dimeglumine (Multihance ${ }^{\circledast}$ ): extended clinical experience from Phase I studies to post-marketing surveillance. J Magn Reson Imaging 2001;14:281-94 CrossRef Medline

12. Shellock FG, Parker JR, Venetianer C, et al. Safety of gadobenate dimeglumine: summary of findings from clinical studies and postmarketing surveillance. Invest Radiol 2006;41:500-09 CrossRef Medline

13. Bleicher AG, Kanal E. Assessment of adverse reaction rates to a newly approved MRI contrast agent: review of 23,553 administrations of gadobenate dimeglumine. AJR Am J Roentgenol 2008;191: W307-11 CrossRef Medline

14. Schneider G, Schürholz H, Kirchin MA, et al. Safety and adverse effects during 24 hours after contrast-enhanced MRI with gadobenate dimeglumine (MultiHance) in children. Pediatr Radiol 2013;43:202-11 CrossRef Medline

15. Shen Y, Goerner FL, Snyder C, et al. T1 relaxivities of gadoliniumbased magnetic resonance contrast agents in human whole blood at 1.5, 3, and 7 T. Invest Radiol 2015;50:330-08 CrossRef Medline

16. Colosimo C, Demaerel P, Tortori-Donati P, et al. Comparison of gadobenate dimeglumine (Gd-BOPTA) with gadopentetate dimeglumine (Gd-DTPA) for enhanced MR imaging of brain and spine tumors in pediatric subjects. Pediatr Radiol 2005;35:501-10 CrossRef Medline

17. Schneider G, Maas R, Schultze Kool L, et al. Low-dose gadobenate dimeglumine versus standard dose gadopentetate dimeglumine for contrast-enhanced magnetic resonance imaging of the liver: an intra-individual crossover comparison. Invest Radiol 2003;38:85-94 CrossRef Medline

18. Nandwana SB, Moreno CC, Osipow MT, et al. Gadobenate dimeglumine administration and nephrogenic systemic fibrosis: is there a real risk in patients with impaired renal function? Radiology 2015;276:741-47 CrossRef Medline

19. Bruce R, Wentland AL, Haemel AK, et al. Incidence of nephrogenic systemic fibrosis using gadobenate dimeglumine in 1423 patients with renal insufficiency compared with gadodiamide. Invest Radiol 2016;51:701-05 CrossRef Medline

20. Schneider GK, Stroeder J, Roditi G, et al. T1 signal measurements in pediatric brain: findings after multiple exposures to gadobenate dimeglumine for imaging of nonneurologic disease. AJNR Am J Neuroradiol 2017;38:1799-806 CrossRef Medline

21. Kinner S, Schubert TB, Bruce RJ, et al. Deep brain nuclei T1 shortening after gadobenate dimeglumine in children: influence of radiation and chemotherapy. AJNR Am J Neuroradiol 2018;39:24-30 CrossRef Medline

22. MultiHance prescribing information in the USA. https://www.accessdata. fda.gov/drugsatfda_docs/label/2018/021357s016s017,021358s015s016lbl. pdf. Accessed September 5, 2019

23. Elster $\mathrm{AD}$, Moody $\mathrm{DM}$, Ball MR, et al. Is Gd-DTPA required for routine cranial MR imaging? Radiology 1989;173:231-38 CrossRef Medline

24. Schwartz GJ, Brion LP, Spitzer A. The use of plasma creatinine concentration for estimating glomerular filtration rate in infants, children, and adolescents. Pediatr Clin North Am 1987;34:571-90 CrossRef Medline

25. Hellerstein S. Fluids and electrolytes: physiology. Pediatr Rev 1993;14:70-79 CrossRef Medline

26. Bhargava R, Hahn G, Hirsch W, et al. Contrast-enhanced magnetic resonance imaging in pediatric patients: review and recommendations for current practice. Magn Reson Insights 2013;6:95-111 CrossRef Medline

27. Raschle N, Zuk J, Ortiz-Mantilla S, et al. Pediatric neuroimaging in early childhood and infancy: challenges and practical guidelines. Ann N Y Acad Sci 2012;1252:43-50 CrossRef Medline

28. Alcorn J, McNamara PJ. Ontogeny of hepatic and renal systemic clearance pathways in infants, Part I. Clin Pharmacokinet 2002;41:959-98 CrossRef Medline

29. Fernandez E, Perez R, Hernandez A, et al. Factors and mechanisms for pharmacokinetic differences between pediatric population and adults. Pharmaceutics 2011;3:53-72 CrossRef Medline

30. Ginsberg G, Hattis D, Sonawane B, et al. Evaluation of child/adult pharmacokinetic differences from a database derived from the therapeutic drug literature. Toxicol Sci 2002;66:185-200 CrossRef Medline

31. Kunze C, Mentzel HJ, Krishnamurthy R, et al. Pharmacokinetics and safety of macrocyclic gadobutrol in children aged younger than 2 years including term newborns in comparison to older populations. Invest Radiol 2016;51:50-57 CrossRef Medline

32. Scala M, Koob M, de Buttet S, et al. A pharmacokinetics, efficacy, and safety study of gadoterate meglumine in pediatric subjects aged younger than 2 years. Invest Radiol 2018;53:70-79 CrossRef Medline

33. Emond S, Brunelle F. Gd-DOTA administration at MRI in children younger than 18 months of age: immediate adverse reactions. Pediatr Radiol 2011;41:1401-06 CrossRef Medline

34. Bhargava R, Noga M. Safety and efficacy of gadobutrol-enhanced MRI in patients aged under 2 years: a single-center, observational study. Magn Reson Insights 2013;6:1-12 CrossRef Medline 
35. Hamm B, Thoeni RF, Gould RG, et al. Focal liver lesions: characterization with nonenhanced and dynamic contrast material-enhanced MR imaging. Radiology 1994;190:417-23 CrossRef Medline

36. Kanal E, Maravilla K, Rowley HA. Gadolinium contrast agents for CNS imaging: current concepts and clinical evidence. AJNR Am J Neuroradiol 2014;35:2215-26 CrossRef Medline

37. Vaneckova M, Herman M, Smith MP, et al. The benefits of high relaxivity for brain tumor imaging: results of a multicenter intraindividual crossover comparison of gadobenate dimeglumine with gadoterate meglumine (The BENEFIT Study). AJNR Am J Neuroradiol 2015;36:1589-98 CrossRef Medline

38. Khouri Chalouhi K, Papini GD, Bandirali M, et al. Less is better? Intraindividual and interindividual comparison between $\mathbf{0 . 0 7 5}$ $\mathrm{mmol} / \mathrm{kg}$ of gadobenate dimeglumine and $0.1 \mathrm{mmol} / \mathrm{kg}$ of gadoterate meglumine for cranial MRI. Eur J Radiol 2014;83:1245-49 CrossRef Medline

39. Huang B, Liang CH, Liu HJ, et al. Low dose contrast-enhanced magnetic resonance imaging of brain metastases at 3.0T using high- relaxivity contrast agents. Acta Radiol 2010;51:78-84 CrossRef Medline

40. Crisi G, Filice S, Erb G, et al. Effectiveness of a high relaxivity contrast agent administered at half dose in dynamic susceptibility contrast MRI of brain gliomas. J Magn Reson Imaging 2017;45:500-06 CrossRef Medline

41. Filice S, Crisi G, Erb G. T2*-correction in dynamic contrastenhanced magnetic resonance imaging of glioblastoma from a half dose of high-relaxivity contrast agent. J Comput Assist Tomogr 2017;41:816-21 CrossRef Medline

42. Murata N, Gonzalez-Cuyar LF, Murata K, et al. Macrocyclic and other non-group 1 gadolinium contrast agents deposit low levels of gadolinium in brain and bone tissue: preliminary results from 9 patients with normal renal function. Invest Radiol 2016;51:447-53 CrossRef Medline

43. Bussi S, Penard L, Bonafè R, et al. Non-clinical assessment of safety and gadolinium deposition after cumulative administration of gadobenate dimeglumine (MultiHance ${ }^{\circ}$ to neonatal and juvenile rats. Regul Toxicol Pharmacol 2018;92:268-27 CrossRef Medline 